\title{
TURÁN INEQUALITIES AND ZEROS OF ORTHOGONAL POLYNOMIALS*
}

\author{
ILIA KRASIKOV $\mathrm{K}^{\dagger}$
}

\begin{abstract}
We use Turán type inequalities to give new non-asymptotic bounds on the extreme zeros of orthogonal polynomials in terms of the coefficients of their three term recurrence. Most of our results deal with symmetric polynomials satisfying the three term recurrence $p_{k+1}=x p_{k}-c_{k} p_{k-1}$, with a nondecreasing sequence $\left\{c_{k}\right\}$. As a special case they include a non-asymptotic version of Máté, Nevai and Totik result on the largest zeros of orthogonal polynomials with $c_{k}=c k^{2 \delta}\left(1+o\left(k^{-2 / 3}\right)\right)$. Our proof is based on new Turán inequalities which are obtained by analogy with higher order Laguerre inequalities.
\end{abstract}

Key words. orthogonal polynomials, Turán inequalities, three term recurrence

AMS subject classifications. $33 \mathrm{C} 45$

1. Introduction. Let $\mathcal{P}=\left\{p_{i}(x)\right\}_{k=-1}^{\infty}$ be a family of orthogonal polynomials satisfying the three term recurrence relation

$$
b_{k} p_{k+1}(x)=\left(x-a_{k}\right) p_{k}(x)-c_{k} p_{k-1}(x), \quad b_{k}, c_{k}>0,
$$

with the initial conditions $p_{-1}=0, p_{0}=1$, and let $x_{1 k}<\ldots<x_{k k}$, be the zeros of $p_{k}(x)$. We are interested in finding uniform bounds on the extreme zeros, that is an interval $I=[A(k), B(k)]$, such that $A(k)<x_{1 k}<x_{k k}<B(k)$, in terms of the coefficients of the recurrence. Such a setting arises naturally if one deals with a family depending on parameters, as in the case of classical Jacobi and Laguerre polynomials, and is seeking for bounds uniform in all the parameters involved. The main aim of this paper is to show that the classical Turán inequality

$$
T_{k}(\mathcal{P}, x)=p_{k}^{2}(x)-p_{k-1}(x) p_{k+1}(x) \geq 0,
$$

and its analogues (abbreviated as TI in the sequel), provide a convenient tool for tackling the problem. It is known that (2) holds for some families of orthogonal polynomials including Laguerre, Jacobi and some other polynomials (see [21] and the references therein).

At present there are two general approaches to the problem, one is based on the chain sequences [8] and another exploiting the Rayleigh quotient to find the extreme eigenvalues of the corresponding Jacobi matrix (see e.g. [6], [7], [15]). The last one yields the following elegant representation for the extreme zeros.

$$
\begin{aligned}
& x_{1 k}=\min \left(\sum_{i=0}^{k-1} a_{i} x_{i}^{2}-2 \sum_{i=0}^{k-2} x_{i} x_{i+1} \sqrt{b_{i} c_{i+1}}\right), \\
& x_{k k}=\max \left(\sum_{i=0}^{k-1} a_{i} x_{i}^{2}+2 \sum_{i=0}^{k-2} x_{i} x_{i+1} \sqrt{b_{i} c_{i+1}}\right),
\end{aligned}
$$

\footnotetext{
${ }^{*}$ Received April 7, 2005; accepted for publication June 24, 2005.

${ }^{\dagger}$ Department of Mathematical Sciences, Brunel University, Uxbridge UB8 3PH, United Kingdom (mastiik@brunel.ac.uk).
} 
where the extrema are taken over all (or only over positive) $x_{0}, x_{1}, \ldots, x_{k-1}$, subjected to $\sum_{i=0}^{k-1} x_{i}^{2}=1$.

For symmetric polynomials (i.e. when $p_{k}(-x)=(-1)^{k} p_{k}(x)$ ) and the monic normalization, the case we mainly deal with in this paper, the recurrence (1) can be rewritten as

$$
p_{k+1}(x)=x p_{k}(x)-c_{k} p_{k-1}(x)
$$

and (3), (4) become

$$
x_{k k}=-x_{1 k}=2 \max \left(\sum_{i=0}^{k-2} x_{i} x_{i+1} \sqrt{c_{i+1}}\right) .
$$

Thus $x_{k k}$ as a function of the vector $\left(c_{1}, c_{2}, \ldots\right)$, possesses many nice properties, e.g. it is clearly subadditive, continuous, increasing with $k$. This makes transparent many otherwise puzzling questions concerning the behaviour of the extreme zeros. The most striking result obtained via (3), (4) is due to A. Máté, P. Nevai and V. Totik [17] and states that if $c, \delta>0$, are fixed and $c_{k}=c^{2} k^{2 \delta}\left(1+o\left(k^{-2 / 3}\right)\right)$, then

$$
x_{k k} k^{-\delta}=2 c-c \cdot 3^{-1 / 3}(2 \delta)^{2 / 3} i_{1} k^{-2 / 3}+o\left(k^{-2 / 3}\right),
$$

where $i_{1}=3.3721 \ldots$, is the smallest zero of the Airy function. This is a far reaching generalization of classical bounds in the Hermite case (see e.g. [20]).

If $x_{k k}$ is represented by a series in decreasing powers of $k$, it is naturally to distinguish between the first and the second order bounds, e.g. $2 c$ and $2 c\left(1-K \delta^{2 / 3} k^{-2 / 3}\right)$ in (7), where to maintain the uniformity in $k$ we will allow a weaker constant $K$ than the exact asymptotic one. Whereas the first order bounds can be obtained rather easily, say, by replacing $2 x_{i} x_{i+1}$ in (6) by $x_{i}^{2}+x_{i+1}^{2}$, or by similar elementary arguments $[2],[15]$, the second order estimates uniform in all the parameters, which are the main subject of this paper, were found only recently for the case of classical orthogonal polynomials [10] - [14].

It is worth also noticing that it is rather easy to extract bounds on the zero $x_{k k}^{*}$ corresponding to the perturbed recurrence $p_{k+1}(x)=x p_{k}(x)-c_{k}\left(1+\epsilon_{k}\right)^{2} p_{k-1}(x)$, provided one knows the result for $\epsilon=0$. Indeed, $\sum_{i=0}^{k-2} x_{i} x_{i+1} \leq 1$, and thus, $x_{k k}^{*}$ is in the interval $(1 \pm \epsilon) x_{k k}$, for $\left|\epsilon_{k}\right|<\epsilon<1$. For example it would be enough to establish (7) for $c_{k}=c^{2} k^{2 \delta}$, the general case with the extra factor $\left(1+o\left(k^{-2 / 3}\right)\right)$, follows from the above arguments. In this paper we will not state explicitly such obvious generalizations, but the reader should keep them in mind.

Our approach to the problem is quite different from the above two and based on the following observation [9]. Let $f=f(x)$ and $g=g(x),|\operatorname{deg}(f)-\operatorname{deg}(g)| \leq 1$, be two real polynomials with only real interlacing zeros. Suppose also that we know a form $\sum_{i=0}^{m} A_{i}(x) f^{m-i} g^{i} \geq 0$, which is indefinite in $f$ and $g$ viewed as formal variables. Then one can routinely obtain bounds on the extreme zeros of $f$ and $g$ similar to (7) but, of course, with a weaker constant instead of $i_{1}$. The existence of such a form is far from being obvious and in fact the main difficulty is to find an appropriate one. For classical orthogonal polynomials, when a second order differential equation is known, one may choose $f=p_{k}, g=p_{k}^{\prime}$, and the quadratic form obtained from the Laguerre inequality $f^{\prime 2}-f f^{\prime \prime}>0$, or its higher order generalizations $[9,10,11,14]$. In the discrete case $f=p_{k}(x+1), g=p_{k}(x)$, play a similar role $[9,12,13]$.

In this paper we will show that the required forms, in particular these giving second 
order bounds, may be obtained directly from $T I$ via the three term recurrence. To this end we will establish two new sets of TI, Theorems 9 and 12 below, yielding second order bounds. The following theorem is one of our main results.

THEOREM 1. Let $p_{k}$ be a polynomial satisfying (5) and suppose that $c_{k}$ are nondecreasing. Then

$$
x_{k k}^{2}<4 c_{k-1}, \quad k \geq 2 .
$$

Moreover if $d_{i}=\frac{c_{i}-c_{i-1}}{c_{i}} \geq 0, c_{0}=0$, satisfy

$$
\frac{d_{i}}{2\left(1+d_{i}\right)}<d_{i+1}<\frac{d_{i}\left(1+2 \sqrt{d_{i}}+2 d_{i}\right)}{1+d_{i}}, \quad i=1,2, \ldots
$$

then for $k \geq 2$,

$$
\begin{gathered}
x_{k k}^{2}< \\
4 c_{k}\left(1-\left(\frac{d_{k+1}}{36}\right)^{2 / 3}\left(\left(\sqrt{6 d_{k+1}+81}+9\right)^{1 / 3}-\left(\sqrt{6 d_{k+1}+81}-9\right)^{1 / 3}\right)^{2}\right)< \\
4 c_{k}\left(1-\frac{d_{k+1}^{2 / 3}}{\left(2^{1 / 3}+d_{k+1}^{1 / 3}\right)^{2}}\right) .
\end{gathered}
$$

Inequality (8) is not new but holds unconditionally and is given here for completeness. In fact slightly better first order bounds $x_{k k} \leq 2 \sqrt{c_{k-1}} \cos \frac{\pi}{k+1}$, are known [8]. As a corollary of Theorem 1 we obtain the following uniform version of (7).

Theorem 2. Let $c_{k}=c^{2} k^{2 \delta}$, where $c>0, \delta \geq 0$, are fixed. Then

$$
x_{k k} k^{-\delta}<2 c \sqrt{1-\frac{\delta^{2 / 3}}{\left(\left(k+\frac{1}{2}\right)^{1 / 3}+\delta^{1 / 3}\right)^{2}}}, \quad k \geq 2 .
$$

It would be important to have lower bounds corresponding to (10). A trivial one $x_{k k}>\sqrt{c_{k-1}}$ readily follows from (6) (see also [2], [14] and Lemma 7 below in this connection). One hardly could expect that (10) is sharp for a faster than polynomial rate of growth of $c_{k}$. It would be also interesting to obtain similar results for polynomials orthogonal on $[-1,1]$ and $[0, \infty)$.

The paper is organized as follows. In the next section we survey some known $T I$ which will be used in section 3 for obtaining bounds on the extreme zeros. In the last section we establish two new sets of $T I$ and give second order bounds for a vast class of orthogonal polynomials satisfying (5) with a nondecreasing sequence $c_{k}$.

2. Turán Inequalities. The reason for study $T I$ in the theory of orthogonal polynomials is that they have a few quite important applications. For example, under appropriate restrictions $T_{k}(\mathcal{P}, x)$ converges uniformly on compact subsets of $(-1,1)$ to $\frac{2 \sqrt{1-x^{2}}}{\pi \alpha^{\prime}(x)}$, where $\alpha^{\prime}(x)$ is the absolutely continuous part of the corresponding orthogonality measure on $[-1,1]$. The existing technique enables one to obtain similar limiting expressions for higher order analogues of $T I$ as well, e.g. the right hand side 
of inequality (18) below tends to $\frac{8\left(1-x^{2}\right)}{\pi^{2}\left(\alpha^{\prime}(x)\right)^{2}}$, see [5] and the references therein.

Today there are two almost independent theories related to $T I$, the first dealing with the validity of (2) for different families of orthogonal polynomials and goes back to the pioneer work of Turán [22]. Another one is motivated by the theory of entire functions, in particular by the Riemann hypothesis. In the last case much more precise higher order generalization of (2) are known, but the orthogonal polynomials must have a generating function of a very special type. A survey of this theory and the relevant references can be found in [3], [4], [5].

Progress in the first direction was recently summarized by R. Szwarc [21], who established the following result.

THEOREM 3. (i) Let $\mathcal{P}$ be a family of symmetric polynomials orthogonal on $[-1,1]$, where three term recurrence (1) is normalized by

$$
b_{k}+c_{k}=1, c_{0}=0, c_{k+1}>0, b_{k}>0,
$$

that is by $p_{k}(1)=1, p_{k}(-1)=(-1)^{k}$. Then

$$
T_{k}(\mathcal{P}, x) \geq 0, \quad|x| \leq 1,
$$

provided one of the following conditions holds

$\left(i_{a}\right) c_{k}$ is nondecreasing and $c_{k} \leq \frac{1}{2}$;

$\left(i_{b}\right) c_{k}$ is nonincreasing and $c_{k} \geq \frac{1}{2}$.

(ii) Let $\mathcal{P}$ be a family of polynomials orthogonal on $[0, \infty)$, which are normalized by $p_{k}(0)=1$, with the three term recurrence

$$
x p_{k}(x)=-b_{k} p_{k+1}(x)+\left(b_{k}+c_{k}\right) p_{k}(x)-c_{k} p_{k-1}(x),
$$

where $b_{0}=1, c_{0}=0, b_{k}>0, c_{k+1}>0$. Suppose $b_{k}$ and $c_{k}$ are nondecreasing, then

$$
T_{k}(\mathcal{P}, x) \geq 0, x \geq 0,
$$

provided one of the following conditions holds

$\left(i i_{a}\right) c_{k} \leq b_{k}, \quad c_{k}-c_{k-1} \geq b_{k}-b_{k-1}$

(iiib) $c_{k} \geq b_{k}, \quad c_{k}-c_{k-1} \leq b_{k}-b_{k-1}$.

R. Szwarc [21] also obtained similar yet rather technical conditions which guarantee the validity of (2) for a general nonsymmetric polynomial with the orthogonality measure supported on $[-1,1]$. For the sake of simplicity we did not state them here. It seems nothing is known in the case of nonsymmetric polynomials orthogonal on the whole real axis. On the other hand the case of symmetric polynomials orthogonal on $(-\infty, \infty)$ is almost trivial and was given in [1].

THEOREM 4. Let $\mathcal{P}$ be a family of orthogonal polynomials satisfying (5) with a nondecreasing sequence $\left\{c_{k}\right\}_{k=1}^{\infty}$. Then $T_{k}(\mathcal{P}, x) \geq 0$.

Proof. The result follows by $T_{0}=1$, and the following easy to check identity

$$
T_{k+1}(\mathcal{P}, x)=c_{k} T_{k}(\mathcal{P}, x)+\left(c_{k+1}-c_{k}\right) p_{k}^{2}(x) .
$$

A few higher order generalization of (2) are known. To state them we shall consider the Laguerre-Pólya class of functions which consists of real polynomials with only real zeros and real entire functions

$$
F(z)=c e^{-\alpha z^{2}+\beta z} z^{r} \prod_{i}\left(1-z / z_{i}\right) e^{z / z_{i}}
$$


where $\alpha \geq 0, c, \beta$, are real, $r$ is a nonnegative integer and $\sum_{i} z_{i}^{-2}$ is convergent.

Suppose now that a family $\mathcal{U}$ of real functions $u_{k}=u_{k}(x), k=0,1, \ldots$, has for some values of $x$ a generating function of the Laguerre-Pólya class,

$$
\sum_{k=0}^{\infty} u_{k} \frac{z^{k}}{k !}=F(z)
$$

An instructive example is provided by the binary Krawtchouk polynomials $u_{k}=$ $K_{k}^{n}(x), n$ is a positive integer, having the generating function

$$
\sum_{i=0}^{\infty} K_{i}^{n}(x) z^{i}=(1-z)^{x}(1+z)^{n-x}
$$

which satisfy $(16)$ for $x=0,1, \ldots, n$. Another examples are given by ultraspherical $C_{k}^{(\lambda)}$, Hermite $H_{k}$, and Laguerre $L_{k}^{(\alpha)}$ polynomials [5], with

$$
\begin{gathered}
u_{k}=\frac{C_{k}^{(\lambda)}(x)}{C_{k}^{(\lambda)}(1)}, \quad \lambda>-\frac{1}{2}, \quad-1 \leq x \leq 1 \\
u_{k}(x)=H_{k}(x), \quad-\infty<x<\infty \\
u_{k}=\frac{L_{k}^{(\alpha)}(x)}{L_{k}^{(\alpha)}(0)}, \quad \alpha>-1, \quad x \geq 0
\end{gathered}
$$

In the following theorem the first part belongs to M. Patrick [19] and the second one to J. Maříc [16] (the extension of it to the whole Laguerre-Pólya class is due to D.K. Dimitrov [5]).

THEOREM 5. For those values of $x$ for which (16) holds

$$
T_{k}^{(m)}(\mathcal{U}, x)=\frac{1}{2} \sum_{j=0}^{2 m}(-1)^{j+m}\left(\begin{array}{c}
2 m \\
j
\end{array}\right) u_{k-m+j} u_{k+m-j} \geq 0, \quad m=0,1, \ldots
$$

and

$$
S_{k}(\mathcal{U}, x)=4\left(u_{k}^{2}-u_{k-1} u_{k+1}\right)\left(u_{k+1}^{2}-u_{k} u_{k+2}\right)-\left(u_{k} u_{k+1}-u_{k-1} u_{k+2}\right)^{2} \geq 0 .
$$

Notice that $T_{k}^{(1)}(\mathcal{U}, x)$ is just $T_{k}(\mathcal{U}, x)$. The inequality (18) can be viewed as a refinement of (2) and is intimately connected with so-called Newton inequalities [18]. To apply (17),(18) to orthogonal polynomials it would be important to to restate the condition for their validity in terms of the coefficients of (1). For $T_{k}^{(2)}$ and recurrence (5) this will be accomplished in the last section. The corresponding question for $S_{k}$ remains open. 
3. Extreme zeros. In this section we give some first order bounds on the extreme zeros which can be deduced from Theorems 3 and 4 . We also show that $S_{k}(\mathcal{U}, x)$ (which gives a fourth degree form), yields second order bounds for Hermite polynomials. The inequality $T_{k}^{(2)} \geq 0$, will be considered in more details in the next section. First, we describe simple geometric arguments which enable one to deduce bounds on the extreme zeros from a given form. Let $p=p(x), q=q(x)$ be two real polynomials, $\operatorname{deg}(p)=k \geq 2, \operatorname{deg}(q)=k-1$, with only real interlacing zeros $x_{1}, \ldots, x_{k}$, and $y_{1}, \ldots, y_{k-1}$, respectively, $x_{i}<y_{i}<x_{i+1}$. Suppose that for $x \in(M, N), M<x_{1}<$ $x_{k}<N$, where $M$ and $N$ can be finite or infinite, there exists a nonnegative form

$$
\sum_{i=0}^{m} A_{i}(x) q^{i} p^{m-i} \geq 0, \quad m \geq 2, \text { even },
$$

where $A_{i}(x)$ are certain functions defined in all the points of $(M, N)$. Introducing the function $t=t(x)=q / p$, we rewrite it as

$$
Q(t, x)=\sum_{i=0}^{m} A_{i}(x) t^{i} \geq 0 .
$$

Since $\lim _{x \rightarrow \pm \infty} t(x)=0$, and the zeros of $p$ and $q$ are interlacing then $t(x)$ consists of two hyperbolic $B_{0}, B_{k}$ and $k-1$ cotangent-shaped decreasing branches $B_{1}, \ldots, B_{k-1}$, where $B_{i}$ is defined for $x_{i}<x<x_{i+1}, x_{0}=-\infty, x_{k+1}=\infty$. A function $t_{0}=t_{0}(x)$ will be called an $(i, j)$ - transversal if

(i) $t_{0}$ is continuous on $[M, N]$,

(ii) $t_{0}$ intersects each of the branches $B_{l}$, of $t$ for $i \leq l \leq j$.

(iii) there is an open interval $I \subset[M, N]$ such that $Q\left(t_{0}, x\right) \leq 0$ iff $x \in[M, N] \backslash I$.

Obviously, if an $(i, j)$-transversal exists then $\left[x_{i+1}, x_{j}\right] \subset I$, and to get bounds on $x_{i+1}, x_{j}$ one needs just to find the extreme roots of the equation $Q\left(t_{0}, x\right)=0$, on $[M, N]$. Note that any continuous function intersects all the cotangent-shaped branches $B_{1}, \ldots, B_{k-1}$, and is, if (iii) holds, a $(1, k-1)$-transversal, thus giving bounds on $x_{2}$ and $x_{k-1}$. For example, $t_{0}=c x$, is a $(0, k)$-transversal for $c>0$, and a $(1, k-1)$-transversal for $c \leq 0$, provided it satisfies (iii). As we will see, in many cases the condition (iii) is automatically fulfilled and moreover, a naive choice of $t_{0}$ as a solution of $\frac{\partial Q(t, x)}{\partial t}=0$, that is as the function providing the minimum to $Q(t, x)$, does work. The situation is especially simple for quadratic forms, the case we mainly exploit here. Then $Q(t, x)=A_{0}+A_{1} t+A_{2} t^{2}$, and one may try $t_{0}=-\frac{A_{1}}{2 A_{2}}$, with $Q\left(t_{0}, x\right)=\frac{4 A_{0} A_{2}-A_{1}^{2}}{4 A_{2}}$.

In the rest of the paper we will use $t=p_{k-1} / p_{k}$, and with one explicitly stated exception, $t_{0}$ will be chosen as a solution of $\frac{\partial Q(t, x)}{\partial t}=0$.

The simplest way to obtain the required quadratic form $Q(t, x)$ for orthogonal polynomials is to express $p_{i-1}, p_{i}$, and $p_{i+1}$ in $T_{i},|k-i| \leq 1$, via $p_{k-1}, p_{k}$ by the three term recurrence. In this case one gets three (slightly different) bounds on the zeros, we present just one of them in the theorem below. But already for $T_{k \pm 2}$, the above expression for $t_{0}$ may have singularities and our arguments are not applicable without certain restrictions on the coefficients (see Lemma 8 below). Using Theorems 3 and 4 to guarantee the corresponding $T I$ we get the following first order bounds.

TheOREM 6. (i) Let $p_{k}$ be a symmetric polynomial satisfying (5) and suppose that $c_{k}$ are nondecreasing. Then

$$
x_{k k}<2 \sqrt{c_{k-1}}, \quad k \geq 2 .
$$


(ii) Let $p_{k}$ be a symmetric polynomial orthogonal on $[-1,1]$ satisfying (1) and (11). Then

$$
\left|x_{i k}\right|<2 \sqrt{b_{k-1} c_{k-1}}
$$

where $i=1, \ldots, k$, if $c_{k}$ is nondecreasing and $c_{k} \leq \frac{1}{2}$; and $i=2, \ldots, k-1$, if $c_{k}$ is nonincreasing and $c_{k} \geq \frac{1}{2}$.

(iii) Let $p_{k}$ be a polynomial orthogonal on $[0, \infty)$ satisfying (13). If $b_{k}$ and $c_{k}$ are nondecreasing, then

$$
\left(\sqrt{b_{k}}-\sqrt{c_{k}}\right)^{2}<x_{2, k}<x_{k, k}<\left(\sqrt{b_{k}}+\sqrt{c_{k}}\right)^{2}
$$

provided $c_{k} \leq b_{k}, \quad c_{k}-c_{k-1} \geq b_{k}-b_{k-1} ;$ and

$$
\left(\sqrt{b_{k}}-\sqrt{c_{k}}\right)^{2}<x_{1, k}<x_{k, k}<\left(\sqrt{b_{k}}+\sqrt{c_{k}}\right)^{2}
$$

provided $c_{k} \geq b_{k}, \quad c_{k}-c_{k-1} \leq b_{k}-b_{k-1}$.

Proof. (i) By (5) we get

$$
Q(t, x)=c_{k-1} p_{k}^{-2} T_{k-1}(\mathcal{P}, x)=1-x t+c_{k-1} t^{2} \geq 0 .
$$

In our case $M=N=\infty$, and $t_{0}=\frac{x}{2 c_{k-1}}$, is clearly a $(0, k)$-transversal. Finally $4 c_{k-1}^{2} Q\left(t_{0}, x\right)=4 c_{k-1}-x^{2}$, and the result follows.

(ii) Observe that $p_{k}$ does not depend on $b_{k}, c_{k}$. Hence we may assume that $b_{k}=$ $b_{k-1}, \quad c_{k}=c_{k-1}$. Substituting $p_{k+1}$ from (1) (in our case $a_{k}=0$ ), we have for $[M, N]=[-1,1]$

$$
Q(t, x)=b_{k} p_{k}^{-2} T_{k}(\mathcal{P}, x)=b_{k}-x t+c_{k} t^{2} \geq 0,
$$

with $t_{0}=\frac{x}{2 c_{k}}$, and $4 b_{k} c_{k} Q\left(t_{0}, x\right)=4 b_{k} c_{k}-x^{2}$. Obviously, $t_{0}$ is a $(1, k-1)$-transversal. Finally, using the normalization $p_{i}(1)=1, p_{i}(-1)=(-1)^{i}$, and $b_{k}+c_{k}=1$, one can check that $t_{0}(-1) \leq t(-1)$, and $t_{0}(1) \geq t(1)$, only if $c_{k} \leq \frac{1}{2}$. Thus, for $c_{k} \leq \frac{1}{2}, t_{0}$ intersects all the branches of $t$ and hence is a $(0, k)$-transversal.

(iii) Substituting $p_{k+1}$ from (13) we obtain with $[M, N]=[0, \infty]$,

$$
b_{k} p_{k}^{-2} T_{k}(\mathcal{P}, x)=b_{k}-\left(b_{k}+c_{k}-x\right) t+c_{k} t^{2} \geq 0 .
$$

This yields $t_{0}=\frac{b_{k}+c_{k}-x}{2 c_{k}}$, which is at least a $(1, k-1)$-transversal, and

$$
4 b_{k} c_{k} \Delta(x)=\left(x-\left(\sqrt{b_{k}}-\sqrt{c_{k}}\right)^{2}\right)\left(\left(\sqrt{b_{k}}+\sqrt{c_{k}}\right)^{2}-x\right) \geq 0 .
$$

Notice that the polynomials here are normalized so that the sign of the leading coefficient of $p_{k}$ is $(-1)^{k}$, and so the branches of $t(x)$ are upside-down in comparison with the previous cases. Thus, to guarantee the intersection of $t_{0}$ with $B_{k}$ one should check

$$
\lim _{x \rightarrow>\infty} t(x)=-1>\lim _{x \rightarrow>\infty} t_{0}(x)=-\infty
$$

hence $t_{0}$ is a $(1, k)$-transversal. On the other hand to be a $(0, k)$-transversal it should satisfy $t_{0}(0)=\frac{b_{k}+c_{k}}{2 c_{k}} \leq p_{k}(0)=1$. This is the case only if $b_{k} \leq c_{k}$. This completes the proof. 
Note that similar bounds can be obtained for nonsymmetric polynomials orthogonal on a finite interval. The corresponding $T I$ are given in [21]. The restrictions we have to impose in cases (ii) and (iii) to obtain bounds on all the zeros reflect the real situation. An easy example is provided by ultraspherical and Laguerre polynomials with small parameters. Roughly speaking, the reason why the obtained bounds exclude one or both extreme zeros is that these zeros are too close to the ends of the interval of orthogonality and the used inequalities do not have enough precision to distinguish between them.

The following Lemma was suggested by one of the referees and together with (8) provides an answer to the following question arising naturally in connection with the above theorem.

Suppose that $p_{k}$ satisfies (5), what is the maximal rate of growth of $c_{k}$ such that

$$
\lim _{k \rightarrow \infty} \frac{x_{k k}}{2 \sqrt{c_{k-1}}}=1 ?
$$

Lemma 7. Suppose that $\frac{c_{k+1}}{c_{k}} \rightarrow 1, c_{k} \rightarrow \infty$ and $c_{k}$ form a nondecreasind sequence, then (21) holds.

Proof. For any $n=n(k), 0 \leq n \leq k-2$, one has by (6),

$$
\begin{gathered}
\frac{x_{k k}}{2} \geq \max \left(\sum_{i=n}^{k-2} x_{i} x_{i+1} \sqrt{c_{i+1}}\right) \geq \sqrt{c_{n+1}} \max \left(\sum_{i=n}^{k-2} x_{i} x_{i+1}\right)= \\
\sqrt{c_{n+1}} \cos \frac{\pi}{k-n+1},
\end{gathered}
$$

where the maximum is taken over all sequences such that $\sum\left|x_{i}\right|^{2} \leq 1$.

On the other hand it is possible to find a sequence $n(k)$ such that $k-n(k) \rightarrow \infty$ and $\frac{c_{k}}{c_{n(k)}} \rightarrow 1$. Then

$$
\frac{x_{k k}}{2 \sqrt{c_{k-1}}} \rightarrow 1
$$

The following lemma provides some additional information in this direction and may be of independent interest.

Lemma 8. Let $p_{k}$ be a symmetric polynomial satisfying (5) and suppose that $\frac{3}{4} c_{k}<c_{k-1} \leq c_{k}$. Then

$$
x_{k k}<2 \sqrt{c_{k-2}}, \quad k \geq 3 .
$$

Proof. We consider

$$
\begin{gathered}
Q(t, x)=c_{k-2} c_{k-1}^{2} p_{k}^{-2} T_{k-2}(\mathcal{P}, x)= \\
\left(c_{k-1}^{2}-\left(c_{k-1}-c_{k-2}\right) x^{2}\right) t^{2}-x\left(2 c_{k-2}-c_{k-1}\right) t+c_{k-2} \geq 0 .
\end{gathered}
$$


In view of Theorem 6 , (i) we can choose $[M, N]=\left[-2 \sqrt{c_{k-1}}, 2 \sqrt{c_{k-1}}\right]$. Then

$$
t_{0}=\frac{x\left(2 c_{k-2}-c_{k-1}\right)}{2\left(c_{k-1}^{2}-\left(c_{k-1}-c_{k-2}\right) x^{2}\right)},
$$

By the assumption $\frac{3}{4} c_{k}<c_{k-1} \leq c_{k}$, therefore $t_{0}$ is a continuous function on $[M, N]$ and thus a $(0, k)$-transversal. Finally

$$
Q\left(t_{0}, x\right)=\frac{\left(4 c_{k-2}-x^{2}\right) c_{k-1}^{2}}{4\left(c_{k-1}^{2}-\left(c_{k-1}-c_{k-2}\right) x^{2}\right)},
$$

and the result follows.

Similar but more involved calculations with $T_{k-3}$ instead of $T_{k-2}$ yield $x_{k k}<$ $2 \sqrt{c_{k-3}}$, provided $\frac{5+\sqrt{5}}{8} c_{k}<c_{k-1} \leq c_{k}$. We omit the details.

Now we will show that using (18), which yields a fourth degree form, one can obtain much sharper second order bounds. We will consider the simplest case of monic Hermite polynomials $H_{k}$ defined by (5) with $c_{k}=k / 2$. The corresponding asymptotic for $x_{k k}$ given by (7) is

$$
x_{k k}=\sqrt{2 k}-2^{-1 / 2} 3^{-1 / 3} i_{1} k^{-1 / 6} \approx \sqrt{2 k}-1.65 \cdot k^{-1 / 6} .
$$

Putting $u_{k}=H_{k}, t=H_{k-1} / H_{k}$, in (18), we get

$$
\begin{gathered}
Q(t, x)=4 S_{k}(\mathcal{P}, x) u_{k}^{-4}=k^{2}\left(2 k-x^{2}\right) t^{4}-2 k x\left(1+4 k-2 x^{2}\right) t^{3}+ \\
\left(4\left(k+x^{2}\right)\left(2 k+1-x^{2}\right)-1\right) t^{2}-4 x\left(4 k+3-2 x^{2}\right) t+4\left(2 k+2-x^{2}\right) \geq 0 .
\end{gathered}
$$

Choosing the same $t_{0}=\frac{x}{k}$, as for the case of the quadratic form given by $T_{k}$ and calculating $Q\left(t_{0}, x\right)$ we get that all the zeros of $p_{k}$ satisfy

$$
8 k^{2}(k+1)-(6 k+1)(2 k+1) x^{2}+(6 k+2) x^{4}-x^{6} \geq 0 .
$$

This equation has only one positive root $x$ which gives the required bound,

$$
x=\frac{\left(m^{2}-1\right)^{2} \sqrt{m^{4}+4 m^{2}+1}}{3 \sqrt{3} m^{3}}=\sqrt{2 k}-2^{-7 / 6} k^{-1 / 6}+O\left(k^{-5 / 6}\right),
$$

where $m=2^{-1 / 6}(\sqrt{27 k+2}+\sqrt{27 k})^{1 / 3}$. Thus we get $2^{-7 / 6} \approx 4 / 9$, instead of 1.65. Notice that the result can be slightly improved by solving the system $Q(t, x)=0, \frac{\partial Q(t, x)}{\partial t}=0$, exactly. This yields $\sqrt{\frac{4 k-3 k^{1 / 3}+1}{2}} \approx \sqrt{2 k}-0.53 k^{-1 / 6}$, we omit the details.

4. Bounds from higher order Turán inequalities. In this section we will consider only the symmetric case (5). For convenience we put $c_{0}=0$. First, we will establish sufficient conditions for the validity of the inequality

$$
T_{k}^{(2)}=T_{k}^{(2)}(\mathcal{P}, x)=3 p_{k}^{2}-4 p_{k-1} p_{k+1}+p_{k-2} p_{k+2} \geq 0,
$$

in terms of the recurrence (5) and derive the corresponding bounds on the extreme zeros. Next, we will show how to modify $T_{k}=T_{k}^{(1)}$, to obtain second order bounds 
for a vast class of nondecreasing sequences $c_{k}$. In particularly, we will prove Theorems 1 and 2 .

TheOREM 9. Let $\left\{c_{k}\right\}_{k=1}^{\infty}$, be a nondecreasing positive sequence such that

$$
c_{k-1}-3 c_{k}+3 c_{k+1}-c_{k+2} \geq 0 .
$$

Then for $k \geq 2$,

$$
T_{k}^{(2)}(\mathcal{P}, x)=3 p_{k}^{2}-4 p_{k-1} p_{k+1}+p_{k-2} p_{k+2} \geq 0 .
$$

Proof. We have the following directly checked identity

$$
T_{k+1}^{(2)}=c_{k-1} T_{k}^{(2)}+\left(c_{k+2}+3 c_{k}-4 c_{k-1}\right) T_{k}+\left(c_{k-1}-3 c_{k}+3 c_{k+1}-c_{k+2}\right) p_{k}^{2} .
$$

Now the result follows by the induction on $k$ and $T_{2}^{(2)}=\left(c_{0}-3 c_{1}+3 c_{2}-c_{3}\right) x^{2}+3 c_{1}^{2}+c_{1} c_{3}>0$.

REMARK 1. If we set $c_{k}=\sum_{i=1}^{k} \delta_{i}$, then the conditions (22) can be rewritten as $\delta_{i} \geq 0, \delta_{i-1}-2 \delta_{i}+\delta_{i+1}<0$, i.e. $\delta_{i}$ should be a nonnegative concave function of $i$.

For orthogonal polynomials Theorem 9 yields

THEOREM 10. Let $c_{k}$ satisfy the conditions (22) of Theorem 9. Suppose also that the following equation

$$
\begin{gathered}
F(x)=x^{6}-2\left(4 c_{k-1}+c_{k}+c_{k+1}\right) x^{4}+\left(16 c_{k-1}^{2}+\left(c_{k}+c_{k+1}\right)^{2}+\right. \\
\left.4 c_{k-1}\left(5 c_{k}+2 c_{k+1}\right)\right) x^{2}-16 c_{k} c_{k-1}\left(3 c_{k-1}+c_{k+1}\right)=0,
\end{gathered}
$$

has only two real roots. Then all the zeros of $p_{k}$ are confined between them.

Proof. Rewriting $T_{k}^{(2)}$ in terms of $t$ we have

$$
\begin{gathered}
Q(t, x)=c_{k-1} p_{k}^{-2} T_{k}^{(2)}= \\
3 c_{k-1}+c_{k+1}-x^{2}-x\left(4 c_{k-1}-c_{k}+c_{k+1}-x^{2}\right) t+c_{k}\left(4 c_{k-1}-x^{2}\right) t^{2} \geq 0 .
\end{gathered}
$$

Clearly, $x^{2}<4 c_{k-1}$, and we can choose $[M, N]=\left[-2 \sqrt{c_{k-1}}, 2 \sqrt{c_{k-1}}\right]$. Now

$$
t_{0}=\frac{x\left(4 c_{k-1}-c_{k}+c_{k+1}-x^{2}\right)}{2 c_{k}\left(4 c_{k-1}-x^{2}\right)},
$$

is a $(0, k)$ - transversal and any zero $x$ satisfies

$$
Q\left(t_{0}, x\right)=-\frac{F(x)}{4 c_{k}\left(4 c_{k-1}-x^{2}\right)}>0,
$$

yielding the required result. $\square$

To show that (25) indeed gives second order bounds we again consider the monic Hermite polynomials $H_{k}(x)$. The conditions of Lemma 9 are fulfilled as $c_{k}=k / 2$. Solving (25) we get

$$
x_{k k}<\sqrt{2 k-\frac{\left(1+(\sqrt{k}+\sqrt{k-1})^{2 / 3}\right)^{2}}{2(\sqrt{k}+\sqrt{k-1})^{2 / 3}}}=\sqrt{2 k-1}-2^{-5 / 3}(2 k-1)^{-1 / 6}+O\left(k^{-5 / 6}\right) .
$$


Now we will establish a new $T I$ which is valid for a vast class of sequences $c_{k}$. Its form is motivated by analogy with the Hermite-Poulain theorem. Namely, one can strengthen, say, Laguerre inequality $f^{\prime 2}-f f^{\prime \prime} \geq 0$, which holds for real polynomials with only real zeros, by the substitution $f+\lambda f^{\prime}$ instead of $f$ for an appropriate value of $\lambda$. This does not affect the inequality as $f+\lambda f^{\prime}$ is again a polynomial with only real zeros. Similar method may be applied in the discrete case as well [12]. In our case we use the following transformation.

Given a family $\mathcal{P}=\left\{p_{k}\right\}_{k=-1}^{\infty}, \quad p_{-1}=0, p_{0}=1$, of orthogonal polynomials satisfying (5), define $\Delta \mathcal{P}=\left\{q_{k}\right\}_{k=0}^{\infty}$, by $q_{k}(x)=p_{k+1}(x)-c_{k} p_{k-1}(x)$. We have the following explicit form

$$
p_{k}^{-2} T_{k}(\Delta \mathcal{P}, x)=c_{k}\left(4 c_{k}-x^{2}\right) t^{2}-x\left(2 c_{k+1}+2 c_{k}-x^{2}\right) t+4 c_{k+1}-x^{2} .
$$

The following identity can be checked directly.

LEMMa 11.

$$
T_{k+1}(\Delta \mathcal{P}, x)=c_{k} T_{k}(\Delta \mathcal{P}, x)+2 c_{k} \mu_{k} T_{k}(\mathcal{P}, x)+G,
$$

where

$$
\begin{gathered}
p_{k}^{-2} G=2 c_{k}^{2}\left(2 c_{k+2}-2 c_{k}-\mu_{k}\right) t^{2}-2 x c_{k}\left(3 c_{k+2}-2 c_{k+1}-c_{k}-\mu_{k}\right) t+ \\
x^{2}\left(2 c_{k+2}-3 c_{k+1}+c_{k}\right)+4 c_{k+1}\left(c_{k+1}-c_{k}\right)-2 c_{k} \mu_{k}, \\
\mu_{k}=2\left(c_{k+1}-c_{k}\right)+\frac{1}{2}\left(\sqrt{c_{k+1}-c_{k}}-\sqrt{2 c_{k+2}-3 c_{k+1}+c_{k}}\right)^{2} .
\end{gathered}
$$

TheOREM 12. Let $\left\{c_{k}\right\}_{k=1}^{\infty}$ be a nondecreasing sequence satisfying for $k=1,2, \ldots$, the following conditions

$$
\begin{gathered}
2 c_{k+2}-3 c_{k+1}+c_{k} \geq 0, \\
\left(c_{k+1}-c_{k}\right)\left(\sqrt{c_{k+1}-c_{k}}+\sqrt{2 c_{k+2}-3 c_{k+1}+c_{k}}\right) \geq \\
\sqrt{c_{k}}\left|c_{k+2}-2 c_{k+1}+c_{k}\right| .
\end{gathered}
$$

Then

$$
T_{k}(\Delta \mathcal{P}, x) \geq 0
$$

Proof. As $T_{k}(\mathcal{P}, x) \geq 0$, by $c_{i+1} \geq c_{i}$, and $T_{1}(\Delta \mathcal{P}, x)=\left(2 c_{2}-3 c_{1}\right) x^{2}+4 c_{1}^{2}>0$, by (26) it is left to show that $G \geq 0$. For, consider

$$
\begin{gathered}
H=p_{k}^{-2} G=2 c_{k}^{2}\left(2 c_{k+2}-2 c_{k}-\mu_{k}\right) t^{2}-2 x c_{k}\left(3 c_{k+2}-2 c_{k+1}-c_{k}-\mu_{k}\right) t+ \\
x^{2}\left(2 c_{k+2}-3 c_{k+1}+c_{k}\right)+4 c_{k+1}\left(c_{k+1}-c_{k}\right)-2 c_{k} \mu_{k},
\end{gathered}
$$

The coefficient at $t^{2}$ is positive, hence it is left to check that the discriminant of this quadratic in $t$ is nonpositive, what yields

$$
c_{k}\left(c_{k+2}-2 c_{k+1}+c_{k}\right)^{2}-\left(c_{k+1}-c_{k}\right)^{2}\left(\sqrt{c_{k+1}-c_{k}}+\sqrt{2 c_{k+2}-3 c_{k+1}+c_{k}}\right)^{2} \leq 0,
$$


and (26), (27) follow.

Practically the conditions of the above theorem are much less restrictive than those of Theorem 9. Yet formally (22) does not follow from (26),(27), as the example $c_{k}=k^{2}+c$, shows.

The conditions (26) and (27) are rather complicated but can be simplified by the substitution $c_{k} / c_{k-1}=1+d_{k}, d_{k}>0$, giving respectively

$$
\begin{gathered}
d_{k+1} \geq \frac{d_{k}}{2\left(1+d_{k}\right)}, \\
d_{k}\left(\sqrt{d_{k}}+\sqrt{2 d_{k} d_{k+1}+2 d_{k+1}-d_{k}}\right) \geq\left|d_{k} d_{k+1}+d_{k+1}-d_{k}\right| .
\end{gathered}
$$

More practical criteria are given in the following Lemma.

Lemma 13. The conditions of Theorem 12 hold if $d_{k}>0$, and

$$
\frac{d_{k}}{2\left(1+d_{k}\right)}<d_{k+1}<\frac{d_{k}\left(1+2 \sqrt{d_{k}}+2 d_{k}\right)}{1+d_{k}}
$$

Proof. Putting in $(29),(30) d_{k+1}=\frac{d_{k}\left(1+2 y+2 y^{2}\right)}{1+d_{k}}, y \geq-1 / 2$, gives

$$
1+2 y+2 y^{2} \geq \frac{1}{2}, \quad 4 d_{k}^{2}(1+y)^{2}\left(d_{k}-y^{2}\right)>0,
$$

and the result follows.

Now we are in the position to prove (10) and thus Theorem 1. This is accomplished in the following two lemmas.

Lemma 14. Suppose that $d_{i}=\frac{c_{i}-c_{i-1}}{c_{i}} \geq 0$, satisfy (29),(30). Then

$$
x_{k k}^{2}<4 c_{k}\left(1-6^{-4 / 3} d_{k+1}^{2 / 3}\left((v+9)^{1 / 3}-(v-9)^{1 / 3}\right)^{2}\right)
$$

where $v=\sqrt{6 d_{k+1}+81}$.

Proof. Let $[M, N]=\left[-2 \sqrt{c_{k}}, 2 \sqrt{c_{k}}\right]$, and consider $Q(t, x)=p_{k}^{-2} T_{k}(\Delta \mathcal{P}, x)$ given by (28). Then we find

$$
t_{0}=\frac{x\left(2 c_{k+1}+2 c_{k}-x^{2}\right)}{2 c_{k}\left(4 c_{k}-x^{2}\right)},
$$

and $t_{0}$ is a $(0, k)$-transversal. Calculating $Q\left(t_{0}, x\right)$ we conclude that all the zeros of $p_{k}$ satisfy

$$
x^{6}-4\left(c_{k+1}+2 c_{k}\right) x^{4}+4\left(c_{k+1}+c_{k}\right)\left(c_{k+1}+5 c_{k}\right) x^{2}-64 c_{k}^{2} c_{k+1}<0
$$

The corresponding equation has only two real roots giving the required bounds, namely

$$
x^{2}=4 c_{k}\left(1-6^{-4 / 3} d_{k+1}^{2 / 3}\left((v+9)^{1 / 3}-(v-9)^{1 / 3}\right)^{2}\right) .
$$


To show that there are no other roots we calculate the discriminant of (32) which is

$$
-2{ }^{28} c_{k+1} c_{k}^{4}\left(c_{k+1}-c_{k}\right)^{8}\left(2 c_{k+1}+25 c_{k}\right)^{2} .
$$

As it does not change the sign, provided $c_{k+1}>c_{k}>0$, the number of real zeros is the same for any such a choice of $c_{k}, c_{k+1}$. Choosing $c_{k}=1, c_{k+1}=2$, we obtain the test equation $x^{6}-16 x^{4}+84 x^{2}-128=0$, having only two real roots.

Lemma 15. With the conditions of Lemma 14

$$
x_{k k}^{2}<4 c_{k}\left(1-\frac{d_{k+1}^{2 / 3}}{\left(2^{1 / 3}+d_{k+1}^{1 / 3}\right)^{2}}\right) .
$$

Proof. It is enough to show that

$$
(v+9)^{1 / 3}-(v-9)^{1 / 3}>\frac{6^{2 / 3}}{2^{1 / 3}+d_{k+1}^{1 / 3}} .
$$

This inequality is transformed into an obvious one, $y>\frac{y}{1+y-y^{3}}$, by the substitution $d_{k+1}=\frac{2\left(1-y^{3}\right)^{3}}{y^{3}}, 0<y \leq 1$.

REMARK 2. The result of Theorem 1 can be strengthened if one expresses $T_{k}(\Delta \mathcal{P}, x)$ in terms of $t^{*}=p_{k} / p_{k+1}$ instead of $t$ as it has been done in Lemma 8. This yields a bound similar to (10) with $c_{k-1}$ and $d_{k}$ instead of $c_{k}$ and $d_{k+1}$ respectively. We don't give the details as the resulting expression is more complicated than (10) and gives only a marginal improvement for $c_{k}$ with less than exponential rate of growth.

Finally, Theorem 2 follows from (33) with $d_{k+1}=\left(1+\frac{1}{k}\right)^{2 \delta}-1$. For we observe that $\frac{d_{k+1}^{1 / 3}}{2^{1 / 3}+d_{k+1}^{1 / 3}}$ is an increasing function in $d_{k+1}$. Now the result follows by applying the elementary inequality

$$
\left(1+\frac{1}{k}\right)^{2 \delta}-1 \geq \frac{2 \delta}{k+\frac{1}{2}}, \quad \delta \geq 0 .
$$

Moreover, $k+\frac{1}{2}$ may be replaced by $k$ for $\delta \geq \frac{1}{2}$.

Acknowledgement. I thank one of the referees not only for Lemma 7 and its proof but also for some other valuable comments.

\section{REFERENCES}

[1] R. Askey, Linearization of the product of orthogonal polynomials, in Problems in Analysis, R. Gunning, ed., Princeton University Press, Princeton, New Jersey, (1970), pp. 223-228.

[2] D. W. LeE, W. VAn Assche, Asymptotic of orthogonal polynomials by three term recurrence relation, preprint.

[3] T. Craven, G. Csordas, Iterated Turán and Laguerre inequalities, J. Ineq. in Pure and Appl. Math. 3, (2003).

[4] T. Craven, G. Csordas, Composition theorems, multiplier sequences and complex zero decreasing sequences, in Value Distribution Theory and Related Topics, Advances in Complex Analysis and Its Applications, Vol. 3, eds. G. Barsegian, I. Laine and C. C. Yang, Kluwer Press, 2004. 
[5] D. K. Dimitrov, Higher order Turán inequalities, Proc. Amer. Math. Soc., 126 (1998), pp. 2033-2037.

[6] G. Freud, On the greatest zero of an orthogonal polynomial, J.Approx. Theory, 46 (1986), pp. $16-24$.

[7] R. A. Horn and C. R. Johnson, Matrix Analysis, Cambridge University Press, 1996.

[8] M. E. H. Ismail And X. Li, Bounds on the extreme zeros of orthogonal polynomials, Proc. Amer. Math. Soc., 115 (1992), pp. 131-140.

[9] I. Krasikov, Nonnegative quadratic forms and bounds on orthogonal polynomials, J. Approx. Theory, 111 (2001), pp. 31-49.

[10] I. Krasikov, Bounds for zeros of the Laguerre polynomials, J. Approx. Theory, 121 (2003), pp. $287-291$.

[11] I. KRAsikov, On zeros of polynomials and allied functions satisfying second order differential equation, East J. Approx., 9 (2003), pp. 51-65.

[12] I. Krasikov, Discrete analogues of the Laguerre inequality, Analysis and Applications, 1 (2003), pp. 189-198.

[13] I. KRAsikov, Bounds for zeros of the Charlier polynomials, Methods and Applications of Analysis, 9 (2002), pp. 599-610.

[14] I. Krasikov, On extreme zeros of classical orthogonal polynomials, J. Comp. Appl. Math., to appear.

[15] V. I. Levenstein, Universal bounds on codes and designs, In: Handbook of Coding Theory, Vol.1, North-Holland, 1998, pp. 499-648.

[16] J. MAŘík, On polynomials with all real zeros, Časopis Pěst. Mat., 89 (1964), pp. 5-9.

[17] A. Máté, P. Nevai, V. Totik, Asymptotic of the zeros of orthogonal polynomials associated with infinite intervals, J. London. Math. Soc., 33 (1986), pp. 303-310.

[18] C. P. Niculescu, A new look at Newton's inequalities, J. Ineq. in Pure and Appl. Math., 1 (2000).

[19] M. L. PATRICK, Extension of inequalities of the Laguerre and Turán type, Pacific J. Math., 44 (1973), pp. 675-682.

[20] G. Szegö, Orthogonal Polynomials, Amer. Math. Soc. Colloq. Publ., v.23, Providence, RI, 1975.

[21] R. Szwarc, Positivity of Turán determinants for orthogonal polynomials, in Harmonic Analysis and Hypergroups, (K.A. Ross et al., ed.) Delhi 1995, Birkhauser, Boston-Basel-Berlin 1997, pp. $165-182$.

[22] P. Turán, On the zeros of the polynomials of Legendre, Časopis Pěst. Mat., 75 (1950), pp. $113-122$ 